\title{
Las manifestaciones de la fuerza ilocucionaria en la prensa escrita*
}

LUIS ARM ANDO BENITTEZ PÉREZ**

luisbenitez@gmail.com

Recepción: 26 de agosto de 2013

Aprobación: 10 de noviembre de 2013

${ }^{*}{ }^{-}$Artículo de investigación científica.

** Magíster en Ciencias de la comunicación; comunicador social y periodista; docente adscrito al centro de investigaciones de la Universidad Cooperativa de Colombia, sede Neiva; miembro del grupo de investigación Voces. Esta investigación se realizó con el apoyo de la Universidad Cooperativa de Colombia y la Asociación de Periodistas del Huila (ASPEHU). 


\title{
Resumen
}

El objetivo de este trabajo fue analizar el tipo de acciones discursivas que se usan en los enunciados que componen los textos noticiosos judiciales. Para lograrlo se analizó un corpus de 131 enunciados empleados como testimonios de las fuentes militares citados en los diarios LaNacón del departamento del Huila, y El Lídar, del departamento de Caquetá durante el año 2011. A partir de la tipología de actos de habla planteada por Jhon Austin, se pudo establecer que cerca de un 34\% de los enunciados constituyen un tipo de acto de habla distinto a relatar, lo cual representa una clara forma de interposición de la acción sobre la descripción, que se supone es la principal función del ejercicio de informar.

Palabras clave: enunciado, acto de habla, fuerza ilocucionaria, pragmática.

\section{Manifestations of illocutionary force in written press}

\begin{abstract}
A bstract
The objective of this study was to analyze the type of discursive actions used in statements from judicial news texts. In order to achieve that, a corpus of 131 statements was analyzed. These statements were used as testimonies from military sources and quoted during 2011 in the newspapers: La Nación, from the department of Huila and El Líder, from the department of Caquetá. Using the typology of speech-acts proposed by John Austin, it was possible to conclude that about 34\% of the statements are part of a speech act with a different purpose than describing. This represents a clear form of insertion of action over description, which is supposed to be the main function of informing.
\end{abstract}

Key words: Statement, speech-act, illocutionary force, pragmatic. 


\section{Les manifestations de la force illocutoire dans la presse écrite}

\section{Résumé}

Le but de ce travail est celui d'analyser le type d'actions discursives qui sont utilisées dans les énoncés qui composent les textes des informations judiciaires. Pour y arriver, on a analysé un corpus de 131 énoncés employés comme des témoignages des sources militaires citées dans les journaux La Nadón du département de Huila, el El Lídar, du département de Caquetá pendant l'an 2011. À partir de la typologie des actes de parole proposée par Jhon Austin, on a pu établir que près de 34\% des énoncés, constituent un type d'acte de parole différent de celui de raconter; ce qui représente une forme claire d'interposition de l'action sur la description, qui est supposée étant la principale fonction de l'exercice d'informer.

Mots clés: Énoncé, acte de parole, forcé illocutoire, pragmatique.

\section{As manifestações da força ilocucionaria na midia escrita}

\section{Resumo}

Este trabalho tem por objetivo analisar o tipo de ações discursivas que se usam nos enunciados que compõem os textos noticiosos judiciais. Para lográ-lo se analisou um corpus de 131 enunciados usados como testemunhas das fontes militares citados nos jornais colombianos, La Nacón do etado doHuila, e El Lider do estado do Caquetá durante 0 ano de 2011. Partindo da tipologia de atos de fala proposto por Jhon Austin, se conseguiu estabelecer que $34 \%$ dos enunciados constituem um tipo de ato da fala distinto de relatar, o qual representa uma clara forma de imposição da ação sobre a descrição, que se supõe é a principal função do exercício de informar.

Palavras chave: Enunciado, ato da fala, força ilocucionaria, pragmática. 


\section{Introducción}

En una noticia de carácter judicial siempre hay una fuente oficial, una militar o una autoridad que actúa como vocero. El enunciador del texto es el periodista, pero la veracidad u objetividad de la nota está sustentada en el enunciado que emita esta fuente oficial. Su testimonio puede variar desde una simple afirmación hasta un veredicto o un juicio de opinión, dependiendo de las circunstancias y el hecho que se describe. Este enunciador realiza su discurso a título institucional y en la mayoría de casos estudiados hace referencia a victorias de la parte oficial, a casos de extorsiones, amenazas, a reportes de operaciones 0 a denuncias.

Cuando la noticia toma mucha relevancia en la esfera nacional, en el testimonio oficial aparece una fuente de mayor rango. La importancia del suceso exige el pronunciamiento de una fuente que tenga la autoridad para dar su punto de vista. Es una cadena de valores que se ha construido en la prensa judicial, a mayor trascendencia tenga el suceso noticioso, más categoría debe tener la fuente oficial.

En un enunciado se desencadenan a su vez una serie de propósitos 0 intenciones que se espera que los destinatarios cumplan, Calsamiglia y Tusón (citadas por Cuvardic, 2008) plantean que las palabras, además de su significado referencial, literal, constituyen una forma de acción intencional, por esto a partir de los anteriores autores se plantea en este artículo que en una noticia de carácter judicial "existen fuerzas semánticas que inciden en la interpretación que los lectores hacen de la información" (p. 103). Por ejemplo, en el caso de la información periodística cabe la reflexión si en los testimonios militares se desea prescribir o persuadir por medio de enunciados; según Patiño (2010), "la primera tiene criterios de aplicación mientras que la segunda no tiene ningún criterio, esto significa que los imperativos no cumplen la función persuasiva" (p. 92). 
Ryszard Kapuscins-ki (2003) planteó que los periodistas trabajan con lo que él consideraba la materia más delicada del mundo: la gente. Es por esto que se afirma que con las palabras se pueden destruir vidas. Ante la importancia de estudiar el efecto que puede causar en la opinión pública toda la información de carácter judicial que los lectores consumen a diario, surgieron los planteamientos ¿qué tipo de acción discursiva se usa en el enunciado del testimonio militar? y ¿cómo pretende incidir esta fuente en el lector?

En la teoría austiniana de los actos de habla se plantea el caso en el cual al decir algo, se hace algo, y además que no todos los enunciados sirven para describir un estado de cosas, y por tal motivo, no sirven para ser definidos de la forma verdadero o falso. A este tipo de actos, como perdonar o prometer Austin denominó expresiones realizativas, que hacen parte de los actos de habla en los cuales la acción se desarrolla a medida que la expresión se lleva a cabo. Austin (2010) planteó: "emitir la expresión es realizar una acción y que esta no se concibe normalmente como el mero decir algo" (p. 51). Estos realizativos tienen la apariencia de un enunciado, pero en el fondo son mucho más que eso, porque llevan consigo el peso de la acción. Por tal motivo, este tipo de expresiones lingüísticas se juzgan de la manera, afortunada o desafortunada, de las cosas que pueden ser y salir mal; por tal motivo Austin calificó esta doctrina como la doctrina de los infortunios (infelicities).

D esde este punto de vista, pronunciar una oración es el acto principal con el cual se cierra un hecho, en el caso de las promesas, es cuando terminamos de hablar que se lleva a cabo el compromiso y con lo cual se dice y se hace algo. Según Alarcón (2008), "los verbos que participan en este tipo de enunciados tienen por característica básica no describir, ni registrar ningún hecho, no son verdaderos, ni falsos y al ser expresados en una oración, la acción se ejecuta" (p. 238). Pero Austin plantea que para poder realizar estas promesas también es necesario que se presenten las circunstancias adecuadas para que el acto sea afortunado y, además, realizado de forma correcta. Estas circunstancias y condiciones deben llevarse a cabo en su totalidad o de lo contrario, la proposición será meramente descriptiva. Un ejemplo de esto es que las personas que hacen parte del acto sean las adecuadas, que exista una convención aceptada por todos, que el acto se lleve a cabo en su totalidad y que las personas tengan los sentimientos correspondientes. De faltar alguna de estas condiciones, dependerá si el acto es un desacierto o un abuso. Por consiguiente, como afirma Patiño (1988): "un uso descuidado de las palabras que se emplean puede dar origen a confusiones conceptuales que impiden la reflexión clara” (p. 67). 
Para Austin existen tres pasos que deben tenerse en cuenta para analizar un acto de habla. El primero es el acto locucionario, aquel que se lleva a cabo cuando decimos algo con cierto sentido dentro de un contexto gramatical correcto. Acto seguido viene el ilocucionario, que es aquel que posee una fuerza convencional, es el acto de aseverar, preguntar, ordenar, suplicar; que se realiza al emitir una locución o enunciado, estos actos poseen una fuerza ilocutiva que resulta ser el efecto que el hablante desea que tengan sus palabras sobre el oyente (Jacobson, 2001), o la comprensión de esa fuerza. Finalmente, los perlocucionarios, que son el resultado del hecho de decir algo, que produce efectos sobre los pensamientos o sentimientos; por ejemplo, prometer.

A partir de esta teoría de los actos de habla, Cuvardic (2008) realizó estudios sobre el contenido de los enunciados en las cartas del lector; a partir de este análisis textual cualitativo, el autor determinó cuáles son los macro actos de habla de cada carta y determinó la intención del autor y su posible efecto perlocucionario. Nolan-Grant (2009), por su parte, analiza el poder de los enunciados en la sociedad del siglo XVIII. Existen también análisis históricos del diálogo (Jucker, Fritz y Lebsanft, 1999) y lingüística textual diacrónica (Fries, 1983). Otro trabajo basado en esta teoría involucra los actos de habla para contrastar el acto de invitar en español e inglés (Ruiz de Zarobe, 2000). En el campo de la literatura cabe destacar un trabajo sobre el lenguaje y la violencia en la novela "Tess of the dúrbervilles realizado por Nishimiura" (2005). Por otra parte, Blanco Salgueiro (2008) toma la metodología austiniana para recoger del diccionario todos los verbos con los que según él realizamos cosas malas, como maldecir, ofender, blasfemar, etc.

Algunas de las investigaciones abordadas a partir de la teoría austiniana son las realizadas en los hospitales para determinar por qué los pacientes latinos no acuden a las citas con médicos angloparlantes en países como Estados Unidos (Jacobson, 2011). También se ha investigado los actos de habla contenidos en la Biblia (Thomas, 2007) y el análisis histórico del discurso (Brinton, 2001). En Colombia es de resaltar el trabajo de Morales \& Cortes (2008) en el que se intentó plantear una teoría sobre la interacción social comunicativa. En el aspecto histórico de la teoría de los actos de habla se han realizado trabajos que apuntan a identificar el origen de los planteamientos filosóficos que la generaron (Patiño, 1988). Por otra parte, recientemente han surgido los estudios históricos, pragmáticos, que buscan identificar si anteriormente se usaban los mismos actos de habla de hoy en día y cómo ha 
sido su evolución, podemos encontrar trabajos en pragmática histórica (Jucker, 1995) y pragmática diacrónica (Arnovick, 1999).

La teoría de los actos de habla ha sido criticada en las últimas decadas básicamente porque se considera que la fuerza ilocucionaria no existe (Cohen, 1964). Por otra parte, Jhon Searle (2007) plantea que el acto locucionario no se puede distinguir del ilocucionario. O tra crítica que surge a la teoría sugiere que el carácter convencional de las ilocuciones no es parámetro para estipular algo sobre un enunciado (Rabossi, 1999).

\section{Metodología}

Se decidió analizar las notas judiciales publicadas durante el año 2011, en los diarios El Líder, de la provincia del Caquetá y La Nación del Huila. El corpus total de enunciados analizados fue de 131 notas. El interés principal fue determinar la herramienta lingǘstica de la fuente oficial para incidir en el lector a partir de un análisis textual cualitativo basado en la teoría de los actos de habla. En una primera etapa se realizó un análisis documental y de contenido. Seguidamente se realizó la reducción, preparación y análisis de datos. Luego, se llevaron a cabo los procedimientos técnicos para analizar los datos en forma de codificación descriptiva y primer nivel de categorización, después codificación axial o relación y segundo nivel de categorización y por último, la codificación selectiva y tercer nivel de categorización.

En su aspecto teórico el trabajo se fundamentó en la teoría austiniana de actos de habla. Esta teoría plantea un primer tipo de acto de habla catalogado como verbos judicativos, aquellos donde se realiza la emisión de un veredicto y que se usan para enjuiciar. En este caso se plantea que la validez de un acto depende de si el mismo es llevado a cabo por una persona con el estatus 0 reconocimiento convencional correspondiente, bien sea un jurado o un árbitro. Sin embargo, dentro de este tipo de juicios también están los que son en forma de cálculo o apreciación, pero que en esencia son un juicio a base de pruebas o razones.

En segundo lugar, están los verbos ejercitativos; la diferencia de esta clase de verbos con los judicativos es bastante fina, ya que solo se usan para ejercer una potestad. Los judicativos se emplean para juzgar algo de una manera determinada, mientras que por los ejercitativos, al contrario, se decide que algo debe ser de tal manera. Por este hecho, los ejercitativos consisten en ejercer potestades. Por ejemplo, votar, ordenar, instar, aconsejar, prevenir, etc. 
En tercer lugar, están les verbos compromisorios, que como el nombre bien lo dice son aquellos verbos que comprometen a la persona que los utiliza a hacer algo, a asumir una obligación o manifestar una intención. A esta categoría pertenecen también aquellos verbos que manifiestan declaraciones o intenciones. Se podrían comparar con el incitar y el ordenar, o con tener pensado, o tener la intención y prometer.

Un cuarto lugar de actos de habla lo componen los verbos comportativos, que son aquellos con los cuales asumimos una actitud, y están relacionados con la reacción que tenemos ante la conducta y fortuna de los demás, o sea, con el comportamiento social. D entro de los más nombrados están el felicitar, dar el pésame y disculpar.

Finalmente, los verbos expositivos son utilizados para exponer una opinión, y entre ellos están: analizo, insisto, defino. Para Austin resulta muy difícil clasificar estos verbos dentro de un marco especial, ya que ellos mismos podrían hacer parte de las anteriores listas, como en el caso de "clasifico" que puede hacer parte de los judicativos.

\section{Resultados}

La brevedad de los testimonios usados en las noticias judiciales permite estudiarlos en su totalidad como un acto de habla individual. En un análisis preliminar se halló que las noticias judiciales corresponden al principal elemento de la prensa en el sur de Colombia, porque a excepción de los meses en los que hubo festividades en los dos departamentos, siempre estuvo presente en las primeras páginas, en total, 102 notas. También se debe resaltar que de los 131 testimonios, 60 fueron de autoridades militares y el resto de autoridades policíacas y voceros oficiales sin nombre ni rango.

El acto de habla que más se encontró en los testimonios fue el expositivo, 80 actos de habla en total como herramienta para relatar sucesos o representar hechos sin emitir juicios, obtener un compromiso o describir un comportamiento. Los verbos expositivos se prestan para manifestar opiniones y emitir comunicados. Ejemplos de verbos expositivos: 
Tabla 1. Ejemplo de noticias con verbos expositivos. Total 80/ 131

\begin{tabular}{|c|c|l|l|l|}
\hline Fecha & D epartamento & \multicolumn{1}{c|}{ Titular } & \multicolumn{1}{c|}{ Cargo } & \multicolumn{1}{c|}{ Testimonio } \\
\hline $\begin{array}{c}\text { 01-12- } \\
2011\end{array}$ & Huila & $\begin{array}{l}\text { Carro bomba } \\
\text { aísla a Vegalarga. }\end{array}$ & $\begin{array}{l}\text { Comandante } \\
\text { de la Novena } \\
\text { Brigada. }\end{array}$ & $\begin{array}{l}\text { Los terroristas proce- } \\
\text { dieron a hurtar el } \\
\text { vehículo en el que se } \\
\text { movilizaba el grupo de } \\
\text { trabajadores encar- } \\
\text { gados de la instalación } \\
\text { del servicio. }\end{array}$ \\
\hline $\begin{array}{c}\text { 13-02- } \\
\text { 2011 }\end{array}$ & Huila & $\begin{array}{l}\text { Policía desactiva } \\
\text { potente carga } \\
\text { explosiva. }\end{array}$ & $\begin{array}{l}\text { Comandante de } \\
\text { la Policía en el } \\
\text { Huila coronel. }\end{array}$ & $\begin{array}{l}\text { Además, tenían adhe- } \\
\text { rida 12 granadas de } \\
\text { fragmentación y 300 } \\
\text { metros de cordón } \\
\text { detonante y deto- } \\
\text { nadores eléctricos. }\end{array}$ \\
\hline $08-12-$ & Caquetá & $\begin{array}{l}\text { Farc utiliza } \\
\text { señuelo humano } \\
\text { para atentar } \\
\text { contra la policía. }\end{array}$ & $\begin{array}{l}\text { Coronel } \\
\text { comandante de de } \\
\text { la Policía del } \\
\text { Caquetá. }\end{array}$ & $\begin{array}{l}\text { Milagrosamente los } \\
\text { uniformados solo } \\
\text { quedaron aturdidos } \\
\text { por la explosión deto- } \\
\text { nada por guerrilleros } \\
\text { de las Farc quienes } \\
\text { activaron la carga } \\
\text { electrónicamente a la } \\
\text { legada de los policías. }\end{array}$ \\
\hline
\end{tabular}

Como se puede ver en los anteriores testimonios, la fuente no es sujeto activo en la acción. El verbo recae en una tercera persona del plural y solo describe su acción; los tres casos son muy similares. Según la teoría de los actos de habla, estos son enunciados constativos que son utilizados para describir un estado de las cosas. En el primer caso, se deja claro que los trabajadores perdieron su vehículo. En el segundo testimonio se evidencia el armamento y la carga explosiva. El tercer testimonio da fe de que los uniformados no sufrieron ningún daño.

En segundo lugar, en este análisis aparecen los verbos judicativos, un total de 34 noticias usaron un testimonio con estas características. Según Austin (1962), "tienen como caso típico el acto de emitir un veredicto, ya sea por un 
jurado, por un arbitro, etc. Pero es menester que sean definitivos; pueden consistir, por ejemplo, en una estimación, en un cálculo o en una apreciación". En definitiva, la función final de un judicativo es emitir un juicio sobre algo.

Tabla 2. Ejemplo de noticias con verbos Judicativos. Total 34/ 131

\begin{tabular}{|c|c|l|l|l|}
\hline Fecha & Departamento & \multicolumn{1}{|c|}{ Titular } & \multicolumn{1}{c|}{ Cargo } & \multicolumn{1}{c|}{ Testimonio } \\
\hline $\begin{array}{c}\text { 03-01- } \\
2011\end{array}$ & Huila & $\begin{array}{l}\text { FARC presionan } \\
\text { pago de "vacuna" } \\
\text { con atentado. }\end{array}$ & $\begin{array}{l}\text { Comandante de } \\
\text { la Policía }\end{array}$ & $\begin{array}{l}\text { Se ha podido esta- } \\
\text { blecer queal parecer los } \\
\text { dueños del almacén } \\
\text { han sido extorsionados. }\end{array}$ \\
\hline $\begin{array}{c}\text { 07-12- } \\
2011\end{array}$ & Huila & $\begin{array}{l}\text { Frustran } \\
\text { atentados de las } \\
\text { FARC. }\end{array}$ & $\begin{array}{l}\text { Comandante de } \\
\text { la Novena } \\
\text { Brigada. }\end{array}$ & $\begin{array}{l}\text { Las trampas mortales } \\
\text { habrían sido sem- } \\
\text { bradas por terroristas } \\
\text { de la Teófilo Forero } \\
\text { parafrenar el avance de } \\
\text { las tropas hacia sus } \\
\text { guaridas. }\end{array}$ \\
\hline $\begin{array}{l}14-01- \\
2011\end{array}$ & Caquetá & $\begin{array}{l}\text { El Culebro estaba } \\
\text { poniendo } \\
\text { granadas en } \\
\text { Neiva. }\end{array}$ & $\begin{array}{l}\text { Sabemos que este } \\
\text { delincuente común } \\
\text { viene delinquiendo con } \\
\text { integrantes del frente } \\
\text { Comandante } \\
\text { la Policía }\end{array}$ & \\
\hline
\end{tabular}

Según los ejemplos anteriores se puede establecer que los tres testimonios son del tipo doypor estableidoqueha carrida Lo importante de este tipo de actos de habla es que los juicios emitidos están construidos sobre pruebas, razones 0 hechos. El primer testimonio es interesante en la medida que se puede construir de manera dyppor establecidb+ que los dueños del almacén han sido extorsionados. Sin embargo, al decir (se ha podido establecer + que al parecer), se está desvirtuando la afirmación, con este enunciado la fuente está dando a entender que no está establecido aún nada, porque al pareer denota que las cosas aún son inciertas. 
El segundo testimonio de este tipo de actos de habla judicativos se puede construir de la forma estimoque.. + las trampas fueron sembradas por terroristas. Este testimonio no puede ser del tipo doy por estableidoporque la presencia del condicional compuesto del indicativo denota más una estimación que una descripción de un juicio. Al decir habna sidbse usa una herramienta muy similar a al pareer, que si bien no son contundentes, sí son juicios que llevan a la opinión pública a hacerse una idea de quién fue el autor del hecho.

El último ejemplo de este tipo de acto de habla que más aparece en los testimonios militares es del tipo doypar etableidb Con este acto se deja claro que el sujeto es un delincuente común, que delinque con un frente guerrillero.

En el tercer lugar de este análisis aparecen los verbos comportativos. Este tipo de actos de habla que poco se encontró en las noticias se relaciona con las actitudes y el comportamiento social del emisor. Ejemplos de estos actos son pedir disculpas, felicitar, elogiar, dar el pésame, maldecir o desafiar. A pesar de que sería poco común hallar ejemplos de fuentes militares pidiendo disculpas, maldiciendo o desafiando dentro de sus testimonios, del total de noticias analizadas se encontraron 7 casos de este tipo.

Los tres ejemplos anteriores de verbos comportativos son bien explícitos: lamentar, invitar y agradecer.

En una cuarta clasificación de los verbos se encuentran los compromisorios. Según Austin (1962), estos actos de habla «tienen como caso típico el prometer o el comprometer de otra manera; ellos lo comprometen a uno a hacer algo, pero incluyen también las declaraciones o anuncios de intención, que no son promesas». En este sentido, los enunciados utilizados por las fuentes oficiales son muy escasos. 
Las manifestaciones de la fuerza ilocucionaria en la prensa escrita

Tabla 3. Ejemplo de noticias con verbos comportativos. Total $7 / 131$

\begin{tabular}{|c|c|c|c|c|}
\hline Fecha & D epartamento & Titular & Cargo & Testimonio \\
\hline $\begin{array}{l}\text { 06-01- } \\
2011\end{array}$ & Huila & $\begin{array}{l}\text { A cerrar filas } \\
\text { contra el } \\
\text { terrorismo. }\end{array}$ & $\begin{array}{l}\text { Comandante de } \\
\text { la Policía. }\end{array}$ & $\begin{array}{l}\text { Lamentamos que haya } \\
\text { ocurrido esa explosión } \\
\text { pero con nuestros } \\
\text { técnicos damos un } \\
\text { parte de tranquilidad de } \\
\text { que se ha desactivado } \\
\text { la segunda carga que } \\
\text { hubiera causado si- } \\
\text { tuaciones drásticas. }\end{array}$ \\
\hline $\begin{array}{l}\text { 02-02- } \\
2011\end{array}$ & Huila & $\begin{array}{l}\text { Simulaba ser de } \\
\text { las FARC para } \\
\text { extorsionar. }\end{array}$ & $\begin{array}{l}\text { Coronel Sub- } \\
\text { comandante } \\
\text { de la Policía } \\
\text { Huila. }\end{array}$ & $\begin{array}{l}\text { Q ueremos invitar a } \\
\text { toda la comunidad } \\
\text { para que se acerque } \\
\text { como estos ciuda- } \\
\text { danos y denuncie este } \\
\text { tipo de hechos. }\end{array}$ \\
\hline $\begin{array}{c}14-07- \\
2011\end{array}$ & Caquetá & $\begin{array}{l}\text { Neutralizado } \\
\text { atentado } \\
\text { explosivo en } \\
\text { Puerto Rico. }\end{array}$ & $\begin{array}{l}\text { Comandante } \\
\text { Operativo de } \\
\text { la Décimo } \\
\text { Segunda } \\
\text { Brigada. }\end{array}$ & $\begin{array}{l}\text { G racias a la infor- } \\
\text { mación entregada por } \\
\text { la comunidad, las } \\
\text { tropas verificaron el } \\
\text { lugardonde estaban los } \\
\text { cilindros, acordo- } \\
\text { naron la zona y pos- } \\
\text { teriormente los deto- } \\
\text { naron con ayuda del } \\
\text { grupo especiales. }\end{array}$ \\
\hline
\end{tabular}

Los casos de verbos compromisorios en el primer testimonio oficial corresponde al tipo expresomi inteniónde El acto de habla realizado concierne al compromiso adquirido por parte del enunciador de pagar una recompensa. Aunque no se puede analizar si este acto de habla es afortunado 0 infortunado, porque no se conocen las circunstancias o el contexto en el que se lleva a cabo, se puede afirmar que el rango de la persona que se compromete es el adecuado y está autorizado para hacerlo. 
Tabla 4. Ejemplo de noticias con verbos compromisorios. Total 4/ 131.

\begin{tabular}{|c|c|c|c|c|}
\hline Fecha & D epartamento & Titular & Cargo & Testimonio \\
\hline $\begin{array}{c}22-03- \\
2011\end{array}$ & Caquetá & $\begin{array}{l}\text { Cae caleta con } \\
\text { cilindros bomba. }\end{array}$ & Coronel. & $\begin{array}{l}\text { Vamos a pagar una } \\
\text { recompensa toda vez } \\
\text { que se informó de } \\
\text { manera oportuna por } \\
\text { parte de un ciudadano } \\
\text { que llamó y nos dio la } \\
\text { información precisa, } \\
\text { del personal extraño en } \\
\text { el sector. }\end{array}$ \\
\hline $\begin{array}{c}05-07- \\
2011\end{array}$ & Huila & $\begin{array}{l}\text { Capturado } \\
\text { "perica" en } \\
\text { Pitalito. }\end{array}$ & $\begin{array}{l}\text { Coronel Jimmy } \\
\text { Luna } \\
\text { comandante de } \\
\text { la policía en el } \\
\text { sur del Huila. }\end{array}$ & $\begin{array}{l}\text { Continuaremos desa- } \\
\text { rrollando operativos } \\
\text { coordinados de for- } \\
\text { ma interinstitucional } \\
\text { con la Fiscalía G eneral } \\
\text { de la Nación y que se } \\
\text { traducen en alla- } \\
\text { namientos, los cualesse } \\
\text { vienen efectuando } \\
\text { desde comienzos del } \\
\text { presente año. }\end{array}$ \\
\hline $\begin{array}{c}18-08- \\
2011\end{array}$ & Huila & $\begin{array}{l}\text { Cae reclutador de } \\
\text { menores de las } \\
\text { Farc. }\end{array}$ & $\begin{array}{l}\text { Comandante de } \\
\text { las Novena } \\
\text { Brigada, general } \\
\text { William Torres } \\
\text { Escalante. }\end{array}$ & $\begin{array}{l}\text { El principal objetivo } \\
\text { de los organismos de } \\
\text { seguridad de estados, } \\
\text { es el de desvertebrar la } \\
\text { red de apoyo de las } \\
\text { Farc. }\end{array}$ \\
\hline
\end{tabular}

El segundo ejemplo de estos actos de habla compromisorios se acerca más al tipo mepropanga Con lo que se compromete la fuente de la manera me propong + continuar desarrollando operativos. Para finalizar, el tercer ejemplo de los actos compromisorios responde al tipo expreso mi intenión de Aunque el testimono está en indefinido se hace una evidente relación con la forma plural donde la fuente está inmersa. De esta manera se puede costruir el acto con la forma de la primera persona del indicativo expresomi intenión de+ desvertebrar la red de apoyo de las Farc. 
Los actos de habla menos utilizados por las fuentes oficiales fueron los ejercitativos. Este tipo de actos "consiste en dar una decisión a favor o en contra de cierta línea de conducta, o abogar por ella" (Austin, 1962). La diferencia de estos actos con los demás es que con ellos se da por establecido que algo debe hacerse de determinada manera, y no que tal cosa seajuzgada de esta otra manera. Es decidir, en vez de juzgar.

Tabla 5. Ejemplo de noticias con verbos ejercitativos. Total 6/131.

\begin{tabular}{|c|c|c|c|c|}
\hline Fecha & D epartamento & Titular & Cargo & Testimonio \\
\hline $\begin{array}{c}14-07- \\
2011\end{array}$ & Caquetá & $\begin{array}{l}\text { Neutralizado } \\
\text { atentado } \\
\text { explosivo en } \\
\text { Puerto Rico. }\end{array}$ & Coronel. & $\begin{array}{l}\text { La situación que de- } \\
\text { nunciamos es la actitud } \\
\text { demencial de la co- } \\
\text { lumna móvil Teófilo } \\
\text { Forero de las Farc, al } \\
\text { instalar estos cilindros } \\
\text { cerca a un lugar de } \\
\text { estudio no miden las } \\
\text { consecuencias de estas } \\
\text { acciones. }\end{array}$ \\
\hline $\begin{array}{c}12-02- \\
2011\end{array}$ & Huila & $\begin{array}{l}\text { FARC habrían } \\
\text { asesinado a } \\
\text { dirigente comunal } \\
\text { en Algeciras. }\end{array}$ & Fuente oficial. & $\begin{array}{l}\text { En el pueblo hay } \\
\text { consternación, nadie } \\
\text { entiende por qué } \\
\text { asesinaron a un líder } \\
\text { que ayudaba a la gente. }\end{array}$ \\
\hline $\begin{array}{c}13-02- \\
2011\end{array}$ & Huila & $\begin{array}{l}\text { Policía desactiva } \\
\text { potente carga } \\
\text { explosiva. }\end{array}$ & $\begin{array}{l}\text { Comandante de } \\
\text { las Novena } \\
\text { Brigada. }\end{array}$ & $\begin{array}{l}\text { Lo fundamental es } \\
\text { destacar laimportancia } \\
\text { de la colaboración } \\
\text { ciudadana quien ha } \\
\text { aportado la infor- } \\
\text { mación a la Policía que } \\
\text { logró neutralizar otra } \\
\text { acción terrorista el cual } \\
\text { tenía presuntamente } \\
\text { como su objetivo la } \\
\text { fuerza pública }\end{array}$ \\
\hline
\end{tabular}


En el primer testimonio se puede construir un acto de habla del tipo rechazo + la actitud demencial de la columna móvil Teófilo Forero de las Farc. Se cambia la primera persona del plural por la primera persona del singular. El segundo testimonio se constuye de la forma rechazo + el acto de asesinar a un líder que ayudaba a la gente. El tercer caso es una manifestación clara de la posición a favor que toma el enunciatario sobre el hecho.

\section{Conclusiones}

Como disciplina, la Lingüística estudia todo lo concerniente al lenguaje humano, esto significa estudiarlo como unidad, o entre las unidades que lo conforman y su relación con los aspectos sociales e históricos. Gracias a esta facultad el ser humano ha podido evolucionar y modificar su entorno. A través del lenguaje el hombre ha desarrollado diversas costumbres, formas de vida, de culturas; por tal razón, Steven Pinker (1994) planteó que el lenguaje representa una ventana a la naturaleza humana. El estudio del lenguaje es, para empezar, un reto intelectual y una actividad fascinante en sí misma, el intento de recomponer y de desentrañar el funcionamiento de un rompecabezas enormemente estructurado y complejo (Hualde, Olarrea, Escobar \& Travis, 2010).

La Pragmática es la disciplina de la Lingüística que estudia el lenguaje en su uso y en la acción dentro de la cual ocurre. D entro de este campo, los elementos extralingüísticos desempeñan un papel importante en la interpretación de cualquier evento comunicativo real, pues existe una distancia entre lo que decimos literalmente y lo que realmente queremos decir (Moya, 2009).

Como se pudo observar, no todos los testimonios emitidos por las fuentes militares en noticias judiciales son usados para fortalecer la descripción del hecho informado. Los testimonios fueron realizados siempre a título institucional a través de actos de habla explícitos. En 51 casos, los enunciados citados en la noticia fueron más allá de la mera descripción y se convirtieron en acciones: 34 lo hicieron en forma de veredicto, 7 en forma de manifestación de actitudes o como forma de comportamiento social, 4 en forma de compromiso o promesa, y 6 con el propósito de abogar por una línea o conducta. 
Estos actos de habla, donde se pone de manifiesto el uso de herramientas persuasivas, dejan en claro que el ejercicio del periodismo sí está siendo manipulado de forma indirecta y está sirviendo como herramienta de manipulación. El desconocimiento, tanto del periodista como del lector, de la fuerza ilocucionaria de ciertos verbos del español genera que en la práctica este ejercicio tenga serias inconsistencias.

Entre los macro actos de habla se identificaron victorias de la parte oficial, casos de extorsiones, amenazas, reportes de operaciones y denuncias. Un interrogante que surge a partir del presente trabajo consiste en establecer cuáles son las características o cuáles fueron las circunstancias que originaron cada uno de los 80 testimonios categorizados como expositivos, o los 51 casos en los que se recurrió a otro tipo de acto de habla. Si bien la mayoría de los casos se usó para relatar sucesos o representar hechos sin emitir juicios, obtener un compromiso o describir un comportamiento, no es tarea fácil identificar los patrones que generaron la elección de determinado acto de habla.

\section{Referencias bibliográficas}

Cuvardic D. (2008). Los actos de habla en las cartas a la columna de la Nación. En: Reista Cienias Soiales (II) pp. 101-112. Costa Rica.

Alarcón M. (2008). Austin y Searle: La relación entre verbos y actos perlocucionarios. En: Literatura yLinguistica $N^{\circ} 19$. pp. 235-250. Santiago de Chile.

Arnovick L. (1999). DiadronicPragnatics Seven casestudiesinEngishilloationarydeudqumet. Amsterdam: John Benjamins.

Austin J. (2010). Cómo hacer cosas con palabras Madrid: Paidós.

Blaco Salgueiro A. (2008). Cómo hacer cosas malas con palabras: actos ilocucionarios hostiles y los fundamentos de la teoría de los actos de habla. En: Revista Hispanoamericana de Filosofía Vol.40.N¹18, pp.3-27. España.

Schiffrin D., Tannen D., \& Hamilton, H. (2001). Historical discaurseanalysis Oxford: Wiley Blackwell. 
Cohen J. (1964). Do illocutionary forces exist? En: Philosqphical quartery. 14 (1964), pp. 118-137. Oxford.

Fleischman S. (1990). Phildogy, linguistics and the discaurse of the medieval text, Speeulum Cambridge: Cambridge University Press.

Fries U. (1983). D iachronic textlinguistics. En: S. Hattori yK. Inoue eds, Procedings of the XIIIth International Congress of Linguistics Tokyo: Tokyo Press.

Gimeno F. (1995). Soidingüística histórica (sigos X-XII). Madrid: Visor y Universidad de Alicante.

G randa G.(1980). Historia social e historia lingüística en Hispanoamérica. (Bahía Blanca: Universidad Nacional del Sur). En: Simposio Intemadional de Lengaa y Literatura Hispánicas pp. 203-215.

Hualde J.I., Olarrea A., Escobar A. M., \& Travis C. E . (2010). Introdución a la lingǘstica hispánica Cambridge: Cambridge University Press.

Jacobson H. (2011). Lo que crees que has dicho no es lo que creen que has dicho. En: Panace@ Vol. 2. $N^{\circ}$ 6. California (Estados Unidos).

Jucker A., Fritz G., Lebsanft F. (1999). Histaical dialogueanalysis Bochum: John Benjamins Publishing Company.

Kapuscinski Ryszard. (2003). Los áno sentidos de periodista Bogotá: Fondo de Cultura Económica.

Morales J., Cortes M. (2008). Lengua matema y disarso en los procesos de interación soial comnicativa Colombia: Universidad Surcolombiana.

Nishimura S. (2005). Languaje, violence and irrevocability: speech acts in tess of the dúrbervilles. En: Studies in theNod volumen $37 \mathrm{~N}^{\circ} 2$. pp. 208-222.

Noland Grand C. (2009). Janes Austen's Speech Acts and Languaged Based Societies. (Baltimore). En: SEL Studies in Engish Literature 1500-1900. pp. 863-878.

Moya P. C. (2009). Aproximación pragmática a los conceptos de acto de habla y de acción comunicativa. En: Desded Jardín deFreud (9). Pp.229 244.

Patiño A. (1988). El acto de prometer en hume y el acto de habla preformativo en Austin. Bogotá. En: Idłasyvalares Nº. Manizales.

Pinker S. (1994). TheLangrage Instint Nueva York: Harper Collins. 
Las manifestaciones de la fuerza ilocucionaria en la prensa escrita

Rabossi E. (1999). Adtos dehabla Valladolid: Trota.

Romaine S. (1982). Sociohistaical Linguistics Cambridge: Cambridge University Press.

Ruiz de Zarobe Y. (2000). La cortesía verbal en inglés: Implicaciones en el acto de habla de la oferta. En: Sintagra V.12. pp. 61-72. España.

Searle J. (2007). Ados de Habla Madrid: Cátedra.

Thomas R. (2007). The Value of Speech Act Theory for Interpretation. En: Masters SeminaryJournal pp.3-21. Sun Valley. 